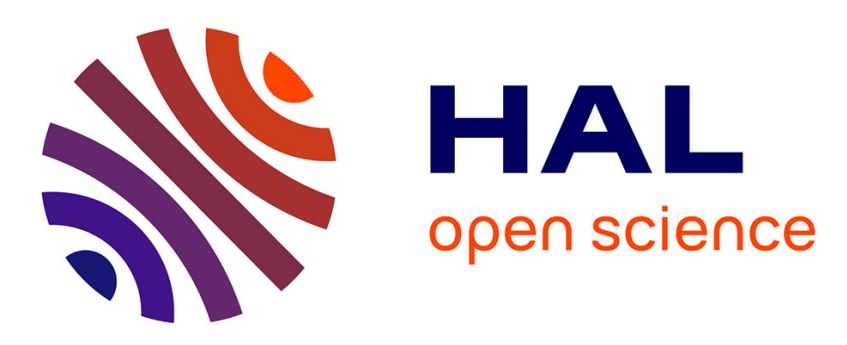

\title{
Solution NMR structure of the SH3 domain of human nephrocystin and analysis of a mutation-causing juvenile nephronophthisis.
}

Albane Le Maire, Thomas Weber, Sophie Saunier, Isabelle Broutin, Corinne Antignac, Arnaud Ducruix, Frédéric Dardel

\section{To cite this version:}

Albane Le Maire, Thomas Weber, Sophie Saunier, Isabelle Broutin, Corinne Antignac, et al.. Solution NMR structure of the SH3 domain of human nephrocystin and analysis of a mutation-causing juvenile nephronophthisis.. Proteins - Structure, Function and Bioinformatics, 2005, 59 (2), pp.347-55. 10.1002/prot.20344 . hal-00106409

\section{HAL Id: hal-00106409 \\ https://hal.science/hal-00106409}

Submitted on 15 Oct 2006

HAL is a multi-disciplinary open access archive for the deposit and dissemination of scientific research documents, whether they are published or not. The documents may come from teaching and research institutions in France or abroad, or from public or private research centers.
L'archive ouverte pluridisciplinaire HAL, est destinée au dépôt et à la diffusion de documents scientifiques de niveau recherche, publiés ou non, émanant des établissements d'enseignement et de recherche français ou étrangers, des laboratoires publics ou privés. 


\title{
Solution NMR structure of the SH3 domain of human nephrocystin and analysis of a mutation causing juvenile nephronophthisis
}

\author{
Albane le Maire ${ }^{1 \S}$, Thomas Weber ${ }^{\S}$, Sophie Saunier ${ }^{2}$, Isabelle \\ Broutin ${ }^{1}$, Corinne Antignac, ${ }^{2,3}$ Arnaud Ducruix ${ }^{1}$ and Frédéric \\ Dardel $^{1 *}$
}

${ }^{1}$ Laboratoire de Cristallographie et RMN Biologiques, UMR8015 CNRS, Faculté de Pharmacie, Université Paris 5, 4 avenue de l'Observatoire, 75006, Paris, France. ${ }^{2}$ Inserm U574, ${ }^{3}$ Service de Génétique, Hôpital Necker-Enfants Malades, Université Paris 5, 75015 Paris, France.

s The first two authors contributed equally to this work.

*Corresponding author : F. Dardel, Cristallographie \& RMN Biologiques, Faculté de Pharmacie, 4 avenue de l'observatoire 75006 Paris, France. Tel: (33) 155739993 ; Fax: (33) 15573 9925 e-mail: frederic.dardel@univ-paris5.fr

Present Adresses : A. le Maire, DIEP, CEA, Saclay, 91190 Gifsur-Yvette, France. Thomas Weber, Henkel, VTB-Enzymtechnologie, Dusseldorf, D-40191, Germany

Short title : nephrocystin SH3 structure

Keywords : NMR; protein folding; cytoskeleton; kidney disease; cell adhesion. 
nephrocystin SH3 structure

\section{ABSTRACT}

Human nephrocystin is a protein associated with juvenile nephronophthisis, an autosomal recessive inherited kidney disease responsible for chronic renal failure in children. It contains an SH3 domain involved in signalling pathways controlling cell adhesion and cytoskeleton organisation. The solution structure of this domain was solved by triple resonance NMR spectroscopy. Within the core, the structure is similar to those previously reported for other sH3 domains, but exhibits a number of specific non-canonical features within the polyproline ligand binding site. Some of the key conserved residues are missing and the $\mathrm{N}$-Src loop exhibits an unusual twisted geometry, which results in a narrowing of the binding groove. This is induced by the replacement of a conserved Asp, Asn or Glu residue by a Pro at one side of the N-Src 1oop. A systematic survey of other sH3 domains also containing a Pro at this position reveals that most of them belong to proteins involved in cell adhesion or motility. A variant of this domain, which carries a point mutation causing nephronophthisis was also analysed. This change, L180P, although it corresponds to a non-conserved and solvent-exposed position, causes a complete loss of the tertiary structure. Similar effects are also observed with the L180A variant. This could be a contextdependent effect resulting from an interaction between neighbouring charged side chains.

Abbreviations : DQF-COSY, Double quantum filtered spectroscopy, GST, Glutathion-S-transferase; HSQC, Heteronuclear single quantum correlation; NOESY, Nuclear Overhauser effect 
nephrocystin SH3 structure

spectroscopy; r.m.s.d., Root mean square deviation; SH3, srchomology 3 domain; TOCSY, Total correlation spectroscopy

\section{INTRODUCTION}

Familial juvenile nephronophtisis (NPH) is an autosomal

recessive and genetically heterogeneous tubulo-interstitial nephropathy responsible for $6-8 \%$ of end stage renal disease in childhood ${ }^{1}$. The first sign of the disease is polyuria, followed by progressive deterioration of renal function during childhood. NPH is characterised by tubular atrophy, abnormal thickening of the tubular basement membrane, interstitial fibrosis, and cyst formation at the cortico-medullary junction. $\mathrm{NPH}$ may be associated with extra-renal manifestations such as retinitis pigmentosa, congenital ocular motor apraxia, liver fibrosis and bone anomalies.

The gene mutated in most patients is NPHP1, coding for the protein nephrocystin, ${ }^{2,3}$ a 732 -amino acid intracellular protein, which exhibits a segmented domain structure: An N-terminal predicted coiled-coil domain, an SH3 domain flanked by two glutamic acid-rich regions, and a highly conserved c-terminal "nephrocystin homology domain" (NHD). NHD bears several functions among which dimer or oligomer formation, epithelial cell-cell junction targeting, interaction with filamins ${ }^{4}$, with the microtubule component beta-tubulin ${ }^{5}$, and with nephrocystin-4, a recently identified protein involved in some cases of juvenile $\mathrm{NPH}{ }^{6,7}$. Nephrocystin, as well as nephrocystin-4 was shown to localize to the cell-cell junctions and to the primary cilia of renal tubular epithelial cells ${ }^{4,5,7}$. 
nephrocystin SH3 structure

The proteins that have been shown to interact with the nephrocystin SH3 domain are implicated in signalling pathways regulating cell adhesion processes and organisation of the cytoskeleton. Among them are p130Cas (Crk-associated substrate ${ }^{4}$ and Pyk $2^{8}$. Therefore, it seems likely that nephrocystin functions as a docking protein that might regulate the organization of the actin and microtubule cytoskeleton and maintain epithelial renal cell polarity ${ }^{4,5}$.

Most of the patients with nephronophthisis have a large deletion in the NPHPI gene ${ }^{9}$. In addition, several point mutations have also been detected, including a leucine to proline change at position 180 within the SH3 domain. The present work addresses the question of nephrocystin SH3 domain structure, as a key to understand the adaptor function of this protein, and of the consequences of the L180P mutation on the protein structure and the onset of the disease. 


\section{MATERIALS AND METHODS}

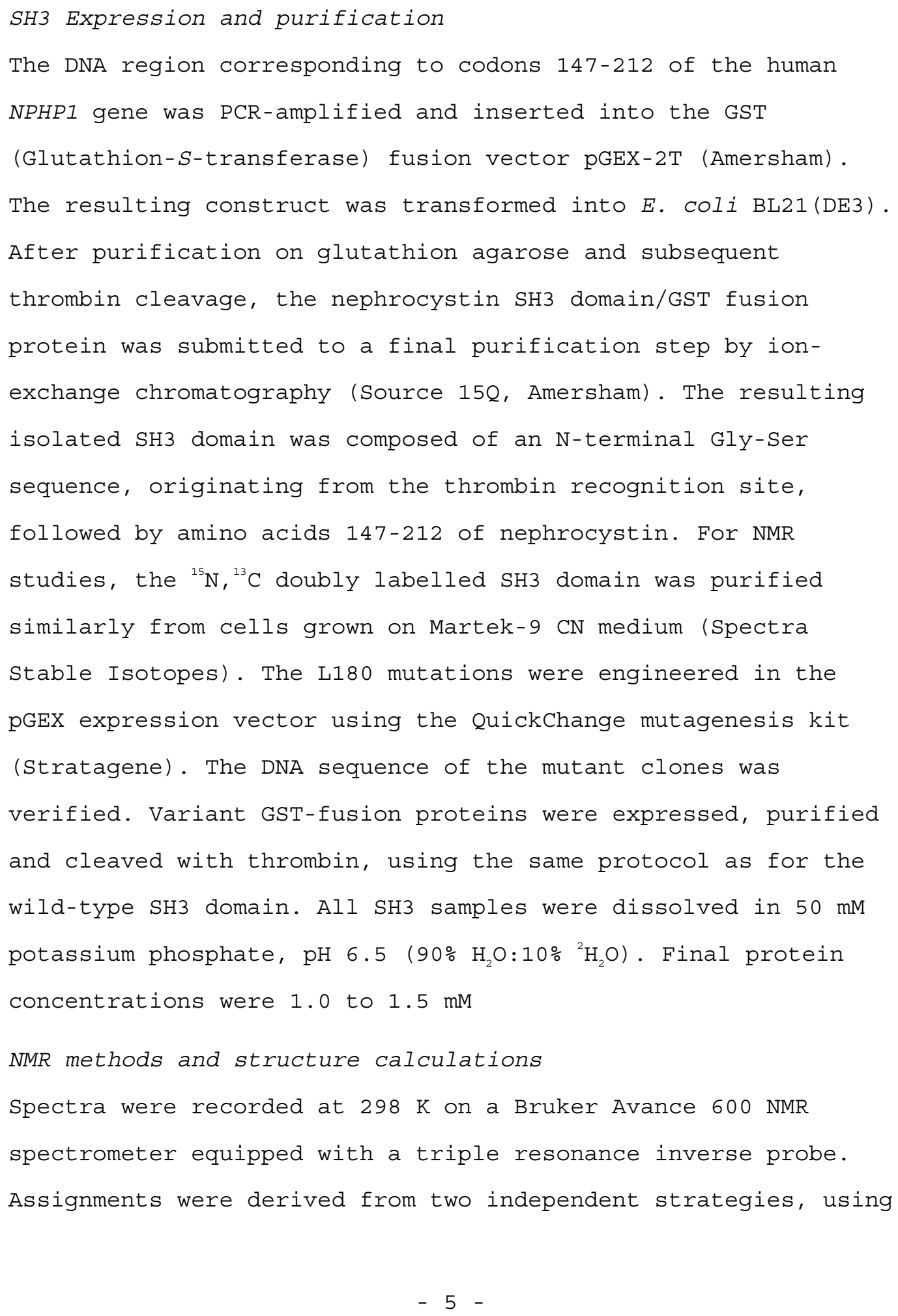


either the 3D HNCACB / CBCA(CO) NH pair of experiments ${ }^{10,11}$ or the 3D ${ }^{15} \mathrm{~N}$-NOESY-HSQC $/{ }^{15} \mathrm{~N}$-TOCSY-HSQC pair of experiments. Owing to the small size of the protein (only 60 observable spin systems), both approaches gave completely consistent sequential assignments, with no ambiguities.

Distance restraints were extracted from either ${ }^{15} \mathrm{~N}$ or ${ }^{13} \mathrm{C}$ NOESYHSQC experiments, both with a mixing time of $150 \mathrm{~ms}$. Distances were classified as strong, medium or weak, and assigned upper limits of $2.5,3.5$ or $5 \AA$, respectively, and a correction of +0.5 ̊ was applied to NOEs involving methyl groups. No ambiguous restraints were used. $\varphi$ dihedral angle restraints were extracted from the analysis of a 3D HNHA experiment ${ }^{12}$. Stereospecific identification of $\beta$-methylene proton and $\chi_{1}$ angles were assigned from the combined analysis of the ${ }^{3} \mathrm{~J}_{\mathrm{N}} \beta$ and ${ }^{3} \mathrm{~J}_{\mathrm{HNH}} \alpha$ extracted from $\mathrm{HNHB}^{13}$ and DQF-COSY experiments, respectively, and from the comparison of the relative intensities of the intra residual $\mathrm{H} \alpha-\mathrm{H} \beta$ and $\mathrm{HN}-\mathrm{H} \beta$ NOE crosspeaks. $\chi_{1}$ angles were constrained to lie within $\pm 60^{\circ}$ of the identified rotamer. Initial conformers were generated with DIANA $^{14}$, using a three stage REDAC strategy ${ }^{15}$ and structures with the lowest target function were refined by restrained simulated annealing using $\mathrm{X}-\mathrm{PLOR}^{16}$, as previously described ${ }^{17}$.

Molecular dynamics

The mutant structure was constructed as follows. The P180 mutation was introduced manually in the PDB file, and the structure was energy minimized in XPLOR, keeping all other atoms fixed and using a purely repulsive van der Waals energy 
nephrocystin SH3 structure

term. The structure was then submitted to two successive short molecular dynamics simulations (2.5 ps each) at $300 \mathrm{~K}$ under the parmallh3x XPLOR forcefield ${ }^{16}$, with the electrostatic term switched off. In the first run, all backbone atoms were constrained to their position in the wild type structure with a harmonic potential of strength $\mathrm{k}_{\text {harm }}=2 \mathrm{kcal} / \mathrm{mol} . / \AA^{2}$, whereas in the subsequent run, this harmonic potential was removed. No major structural changes were observed after this procedure. A final molecular dynamics run (4 ps) was then performed using the full potential, including electrostatics. Solvent shielding was crudely simulated by having a dielectric constant increasing linearly with distance (《 rdie » option in XPLOR). The resulting mutant structure was finally energy minimized. 


\section{RESULTS AND DISCUSSION}

NMR assignment structure of the nephrocystin SH3 domain

The SH3 domain from human nephrocystin was cloned, expressed

and purified as described under materials and methods. Using a doubly ${ }^{15} \mathrm{~N},{ }^{13} \mathrm{C}$ labelled sample, backbone proton, carbon and nitrogen NMR assignments were obtained using standard triple resonance experiments. Chain tracing was straightforward and allowed the unambiguous identification of residues T153 to E212 (sequence shown in figure 1 ). The backbone amide groups of the eight first residues, including the exogeneous Gly-Ser sequence, could not be detected in HSQC type experiments, as they presumably exchanged too fast with the solvent and are most likely disordered. Assignments of backbone amide groups are shown in figure 1. Side chain resonances where identified from the combined analysis of HCCH-TOCSY ${ }^{18}$ and ${ }^{13} \mathrm{C}$ TOCSY-HSQC experiments ${ }^{19}$. Proton assignments were essentially complete, with the exception of part of the side chains of E155, K184,W189, R204 and E212. Stereospecific assignments were obtained for $16 \beta$-methylene protons and for all four valine methyl groups, by the combined analysis of $\mathrm{HNHB},{ }^{15} \mathrm{~N}$ and ${ }^{13} \mathrm{C}$ NOESY-HSQC experiments ${ }^{20}$. In establishing the structure, only residues 153 to 212 where considered. Experimental distance restraints were extracted from the analysis of heteronuclear NOESY experiments and $\varphi$ and $\chi_{1}$ dihedral angles from the analysis of coupling constants and relative intra-residue NOE intensities. Finally, hydrogen bond restraints were included for slowly exchanging amide groups 
which were involved in regular secondary structure elements. Overall, they consisted of 540 NOE-derived restraints (110 intra-residue, 121 sequential, and 309 medium to long range restraints), $32 \varphi$ and $21 \chi_{1}$ angle restraints and 30 hydrogen bond restraints. This corresponded to an average of 10.4 constraints per residue.

The structure of nephrocystin SH3 domain was calculated by a hybrid method combining initial structure generation in torsion angle space with the DIANA program ${ }^{14}$, followed by refinement with $\mathrm{XPLOR}^{16}$, as previously described ${ }^{17}$. Two hundred initial structures were generated using DIANA and the 20 conformers with the lowest target function were retained for refinement with XPLOR. After the restrained simulated annealing stage, three of the resulting structures exhibited a high total energy and strong violations of several experimental restraints and were thus discarded. The final set of converged structures thus contains 17 conformers (PDB entry 1S1N), overlayed in figure 2. All of the 17 showed a correct stereochemistry, a good Van der Waals geometry and satisfied the experimental restraints (see Table I). The r.m.s.d. over the entire set is $0.63 \AA$ for backbone atoms and $1.09 \AA$ for all heavy atoms. If the more disordered parts of the RT-Src and distal loops (residues 166168 and 195-197), as well as the first and the last two residues are excluded, then, the r.m.s.d drops to $0.46 \AA$ for backbone atoms and $0.88 \AA$ for all heavy atoms. Comparison with the structure of other SH3 domains The amino acid sequence of nephrocystin SH3 domain is substantially different from other SH3 domains with known 3D 
nephrocystin SH3 structure

structure, with sequence identity levels ranging from 20 to $40 \%$. The closest match is with the first of the two c-Crk SH3 (40\% sequence identity). Nevertheless, the overall structure of nephrocystin SH3 domain is very similar to the other SH3 structures available in the Protein Data Bank (http://www.rcsb.org/pdb/). When superimposed with the crystal structure of the related c-Crk SH3 (PDB entry 1CKA ${ }^{21}$ ), the r.m.s.d. for all backbone atoms is $1.50 \AA$. The major differences between the two structures are located at the very tip of the RT-SrC and N-SrC loops (Figure 3). If these loop residues are removed from the computation, then the backbone r.m.s.d. drop to $0.79 \AA$, over 45 residues. Within the RT-loop, differences correspond to a small global outward movement of the peptide backbone, with only minor local changes. The slightly more "closed" conformation of the C-Crk SH3 RT-loop could result from the presence of a bound peptide in the corresponding crystal structure. On the other hand, the conformation of the N-SrC loop is quite different in the two structures, with the nephrocystin loop exhibiting a twisting "S-shaped" conformation around residues 185 and 186 (figure 3). This difference in conformation is unambiguously supported by numerous NOEs, in particular involving the side chain of K185, which contacts those of $\mathrm{W} 190$ and T205. In C-Crk, the side chain of the residue corresponding to K185 (N94) points toward the opposite direction, with its $C \gamma$ atom more than $10 \AA$ away from the residues corresponding to W190 and T205. The side chain of K185 is indeed unusually well defined and, because of its close proximity with 190 indole ring, it exhibits strongly 
ring-current shifted $\gamma \mathrm{CH}_{2}$ resonances at -0.33 and $-0.14 \mathrm{ppm}$ (the two rightmost resonances, resolved in the 1D spectrum shown in figure 4).

Specific sequence features of nephrocystin sH3 domain The primary sequence of the nephrocystin SH3 domain exhibits a few significant deviations from the consensus SH3 sequence ${ }^{22}$, all located within the ligand recognition site (shown in figure 2, bottom). In order to investigate possible correlations between those and the specific structural features of nephrocystin SH3, a systematic sequence survey was performed. To this purpose, an extensive set of 1626 SH3 sequences was extracted from the Pfam database (http://www.sanger.ac.uk/Software/Pfam/, accession number PF00018). The first significant unusual feature of the nephrocystin SH3 ligand binding site is the presence of a glycine at position 161 (figure 2, bottom). This corresponds to position 8 in the standard SH3 numbering and is an aromatic residue in $88 \%$ of $\mathrm{SH} 3$ sequences (mostly $\mathrm{Y}$ or $\mathrm{F}$ ). Its aromatic ring forms the "'floor" of the $\mathrm{P}_{2}$ and $\mathrm{P}_{3}$ subsites ${ }^{23}$. Substitution of this residue by a glycine opens a cleft on the surface of the nephrocystin SH3 which could either allow for the binding of a bulkier side chain or accommodate a bending of the ligand backbone. This feature is highly specific, as only 8 of the 1626 examined SH3 domains carried a glycine at this position, half of which correspond to the various available nephrocystin sequences.

The other major unusual sequence feature of the nephrocystin SH3 domain is located within the N-Src loop of nephrocystin SH3 
nephrocystin SH3 structure

and correlates with the unusual conformation of this loop : it has a proline at position 186 (numbered 33 in the SH3 standard nomenclature ${ }^{22}$ ) which induces the observed kink in the backbone (figure 3). In $64 \%$ of the analysed SH3 domains, at this position, either a Glu, Asp or Asn residue is normally found, making side chain contacts with the bound peptide ${ }^{22}$. In nephrocystin, the modified conformation of the N-Src loop narrows the ligand binding groove at the level of the $\mathrm{P}_{-2}$ and $\mathrm{P}_{-4}$ subsites ${ }^{23}$ and hence favours the binding of sequences with smaller side chains at either or both of these positions. Within the 1626 analysed SH3 sequences, 55 contained a proline which could be unambiguously aligned at this position. Among those, nephrocystin is currently the only one for which a 3D structure is available, but it is reasonable to assume that a number of those also exhibit the same twisted conformation of the N-Src loop induced by the proline residue. It was of interest to see whether this particular sequence feature within a key ligand recognition element of the SH3 could correlate with some functional feature of the corresponding proteins, via one or more specific targets of this protein interaction domain. A database search revealed that 43 of these 55 SH3 belong to proteins with either identified or tentatively assigned functions. Interestingly, among those 43 proteins, 40 are either directly or indirectly associated with the cytoskeleton and motility or adhesion : 5 nephrocystins, 9 type I myosins, 7 intersectins (involved in endocytosis, interact with dynamin and N-WASP via their SH3 domains ${ }^{24}$ ), 4 phospholipases C- $\gamma$ (co-localise with actin via their SH3 
nephrocystin SH3 structure

domains ${ }^{25}$ ), 3 Fish (scaffolding proteins localized in actin-rich structures in Src-transformed cells ${ }^{26}$ ), 2 P-dlg (homolog of Drosophila Discs-large protein, involved in cytoskeleton organisation, synaptic junctions and cell polarity maintenance ${ }^{27}$ ), 9 Nox proteins (phagocyte NADPH oxidase activation, these proteins have to be initially translocated to the site of phagocytosis at the plasma membrane, a process involving actin) and yeast RVS167 protein (involved in the regulation of actin distribution, cell polarity, morphology and budding ${ }^{28}$ ). This suggest that possibly, all these proteins can be addressed to active regions of the cytoskeleton via the interaction of their "atypical" SH3 binding groove to one or more specific partners within the actin complex.

The L180P mutation which causes nephronophtisis, strongly destabilises the nephrocystin SH3 structure One patient mutation causing nephronophtisis, L180P, has been mapped into nephrocystin SH3 domain ${ }^{6}$. It corresponds to position 27 in the systematic SH3 sequence alignment ${ }^{22}$, points toward the solvent and is neither part of the extensively characterised structural core nor located within the ligand recognition site. In order to assess the consequences of this mutation on the nephrocystin SH3 domain structure, the corresponding mutant protein was engineered by site-directed mutagenesis and expressed and purified as the wild-type. $1 \mathrm{D}{ }^{1} \mathrm{H}$ NMR data recorded on the L180P SH3 domain protein showed a dramatic change compared to the wild-type protein (figure 4). The mutant domain spectrum corresponds to that of an unfolded 
nephrocystin SH3 structure

protein, showing a very narrow amide spectral width, a

coalescence of aliphatic resonances and no shifted methyl

resonances. Accordingly, the 2D-NOESY spectrum was totally

devoid of signals characteristic of protein tertiary structure,

such as amide-amide contacts, or aromatic-methyl contact (not

shown). Most backbone amide groups showed negative or vanishing

heteronuclear ${ }^{1} \mathrm{H}-{ }^{15} \mathrm{~N}$ NOEs (not shown) which is clearly

indicative of very rapid motions of the backbone, as expected

from an unfolded protein.

Thus, the L180P mutation appears to completely destabilise the

SH3 domain fold. Given the modular organisation of

nephrocystin, it is therefore likely that it also holds in the

context of the full-length protein. The mutant protein will

therefore be unable to interact with its partners, such as p130Cas and Pyk2, and will possibly be more vulnerable to proteasome degradation, thereby explaining the loss of nephrocystin function.

The observation that this mutation completely abolishes formation of the otherwise robust SH3 domain fold is quite surprising, since it corresponds to a non-conserved, solventexposed position. L180 is however located within a $\beta$-strand and substitution by a proline residue might not be tolerated within the corresponding $\beta$-sheet. Accordingly, in the extensive alignment of 1623 SH3 domains available in the Pfam database, this position is quite variable, but never corresponds to a proline. In order to assess whether the observed effect was the result of the insertion of the proline within the $\beta$-strand or of the leucine side chain removal, a "milder" L180A change 
was also engineered and the corresponding SH3 variant was expressed and purified similarly. Most strikingly, its $1 \mathrm{D}{ }^{1} \mathrm{H}$ NMR spectrum exhibited an intermediate behaviour (figure 4), and can be interpreted as a mixture of a minor fraction of the folded wild-type SH3 and a major fraction of the unfolded state, similar to L180P mutant. This shows that, by itself, the leucine side chain at position 180 plays some critical role in the folding and/or the stability of the nephrocystin SH3 fold. To our knowledge, such a dramatic effect of a surface residue on the stability of SH3 domains has never been reported. Indeed, although numerous thorough studies have been performed on the SH3 model system for protein folding ${ }^{29-33}$, most of those have so far been focused on residues forming the conserved structural core, either via their involvement in the hydrophobic core packing or through their contributions to the hydrogen bonding network.

In order to investigate the L180P loss of structure effects, the consequences of this structural change were analysed by molecular modelling. After hand-constructing the mutation into the wild-type coordinates, the resulting structure was regularised with X-PLOR by performing a short molecular dynamics refinement followed by an energy minimisation, in the absence of electrostatics. At this stage, no steric clashes remained and the energy score was essentially identical to that obtained with the wild-type sequence. This indicated that the proline could in principle be quite easily accommodated within the scaffold of the native protein, with only marginal local changes to the structure. The fact that the L180A mutation also destabilised the SH3 was even more surprising, given that, in 
nephrocystin SH3 structure

that case, steric constraints cannot explain the effect and that, in some SH3 sequences, an alanine can be found at this position. This suggested that specific local environment of L180 in nephrocystin might contribute to this dramatic effect. Indeed, examination of the structure revealed that L180 is sandwiched between two oppositely charged residues, E156 and K193, located on the neighbouring $\beta$-strands. Thus, the bulky aliphatic side chain of L180 could act as an electrostatic "insulator" on the surface of nephrocystin SH3 (figure 5, left). In order to assess the influence of these charged residues on the stability of the SH3 fold, we performed a short molecular dynamics simulation of the L180P mutant model constructed above, but including the electrostatic term of the $\mathrm{X}-\mathrm{PLOR}$ energy function. Although this was only a "crude" simulation, performed in vacuo, it does demonstrate that it is sterically possible for the two charged residues to come in close contact over the pyrrolidine ring of P180 and also most likely in the case of an even smaller side chain such as alanine, for the L180A mutant. This hypothesis is compatible with database analysis : within the 1626 SH3 sequences in Pfam, there are 58 other sequences which have a similar pattern with an acidic and a basic at the positions corresponding to E156 and K193, respectively. The intervening residue has a rather bulky side chain in a large majority of cases, with Ile, Leu, Thr and Trp representing 90\% of the observed residues at that position (compared to only $34 \%$ for the whole SH3 set). There is one single exception which carries an intervening Glycine between the two charged residue (entry Q7PYD8), corresponding 
to an undocumented predicted gene from Anopheles Gambiae whole genome sequence.

The L180P mutation could thus destabilise the SH3 fold by three non-exclusive mechanisms : (i) In the absence of a bulky insulating side chain, the movement of solvent-exposed E156 and K193 could twist the attached $\beta$-strands and perturb the delicate packing of the conserved hydrophobic core residues located on the opposite side of the $\beta$-sheet (L179, V181) thereby destabilising the overall folded structure. Indeed the residue equivalent to L179 in Fyn SH3, F26, has been shown to play an important role in the cooperativity of the folding ${ }^{34}$. (ii) The L180P mutation is located very close to the diverging type II $\beta$-turn (residues 173-179 in nephrocystin SH3) which has been shown to be able to fold independently ${ }^{35}$ and proposed to be a nucleation site for $\mathrm{SH} 3$ domain folding ${ }^{36}$. The mutation and/or the electrostatic interaction between E156 and K193 could kinetically interfere with the folding process and/or inhibit the standard folding pathway. (iii) P180 lacks the backbone amide proton which contributes to the stability of the $\beta$-barrel structure by making a hydrogen bond with carbonyl group of adjacent K193. The first two mechanisms also hold for the L180A variant and could thus explain the significant although less severe loss of stability observed for this variant. Overall, this suggests that the drastic destabilisation of the nephrocystin SH3 domain by the L180P or L180A mutations is a context-dependent effect. This is further supported by the observation that in a number of sequences, an alanine residue can be accommodated at this position, as for 
nephrocystin SH3 structure

instance in Pex13P SH3 domain structure (PDB $1 \mathrm{NM} 7^{37}$ ). But in those instances, the alanine is not located between ion-pair forming residues, such as E156 and K193 in nephrocystin.

This study is the first to report the structure of an SH3 domain of a protein involved in an inherited kidney disease. It reveals novel structural features within the ligand binding groove which will have important consequences for its recognition specificity and possibly for its targeting to cytoskeleton proteins. It clearly proves the pathogenic role of a missense mutation, L180P, found in a patient with juvenile NPH. Similar approaches could be further used to test other mutations in the nephrocystin SH3 domain, as well as to study the interaction of nephrocystin with potential SH3 ligands. 


\section{AKNOWLEDGEMENTS}

This work was supported by the Institut National de la Santé et de la Recherche Médicale (INSERM), the Association pour l'Utilisation du Rein Artificiel (AURA). 


\section{LEGEND TO THE FIGURES}

\section{Figure 1 : Sequence and NMR assignment of nephrocystin SH3} domain.

Top : primary sequence of the recombinant nephrocystin SH3 domain used for solution NMR studies. The amino acid numbers correspond to the whole length nephrocystin. The first two residues (GS) orginate from the thrombin cleavage site. Underlined is the SH3 domain sequence. These residues correspond to those which gave detectable signals in amidebased experiments.

Bottom : Assignment of backbone amide groups of nephrocystin $\mathrm{SH} 3$ domain. Shown is a ${ }^{15} \mathrm{~N}$ HSQC spectrum recorded on a $1.5 \mathrm{mM}$ ${ }^{15} \mathrm{~N} ;{ }^{13} \mathrm{C}$ doubly labelled sample dissolved in $50 \mathrm{mM}$ potassium phosphate, pH $6.5\left(90 \% \mathrm{H}_{2} \mathrm{O}: 10 \%{ }^{2} \mathrm{H}_{2} \mathrm{O}\right)$.

\section{Figure 2 : 3D Structure of nephrocystin SH3 domain}

Top : Superimposition of the 17 refined conformers of nephrocystin SH3 domain (PDB entry 1S1N). The backbone is shown in green. Selected well-defined side chains have been indicated. Conserved hydrophobic core residues are shown in red and other residues are shown in blue.

Bottom : Ligand binding site of nephrocystin SH3 domain. Residues which are conserved or semi-conserved in most SH3 sequences are shown in green. Residues which differ from the conserved SH3 consensus are shown in red. Schematic drawing generated with MOLMOL ${ }^{38}$. 
Figure 3 : The N-src loop of nephrocystin SH3 is twisted by a non-canonical proline residue.

Stereo view of the superimposed backbones of nephrocystin (green) and N-Crk SH3 (yellow; PDB: 1CKA). The two structures are very similar, with the exception of the N-Src loop, at the right. Proline 186, which induces a kink in nephrocystin, is shown in red. It corresponds to a position were a semiconserved Glu, Asp or Asn residue is found in most SH3 domains.

Figure 4 : Folding of nephrocystin SH3 variants at position 180 Shown are 1D ${ }^{1} \mathrm{H}$ NMR spectra of wild-type (bottom), L180A (center) and L180P (top) nephrocystin SH3 domain (50 mM Kphosphate $\mathrm{pH} 6.5,293 \mathrm{~K})$. The shaded bars indicate the random coil regions of aliphatic and backbone amide protons, whereas the boxed areas indicate the shifted methyl and backbone amide resonance regions, characteristic of protein tertiary structure.

\section{Figure 5 : Destabilisation of the SH3 fold by the L180} mutations.

Left : Sructure of the wild-type nephrocystin sH3. The side chains of E156, L180 and K193 are indicated. Right : Model of the mutant L180P structure. The diverging type II $\beta$-turn which fold independently in solution is highligthed in yellow. After the molecular dynamics simulation, the major structural change was the movement of E156 and K193 side chains, the terminal atoms of which come in van der Waals contact over the 
nephrocystin SH3 structure

pyrrolidine ring of $\mathrm{P} 180$ (right panel). This also induced a partial disruption of the underlying $\beta$-sheet structure. 


\section{REFERENCES}

1. Salomon R, Gubler MC, Antignac C. Nephronophthisis. in Oxford Textbook of Clinical Nephrology. Third Edition, Oxford University Press, 2004. in press

2. Hildebrandt F, Otto E, Rensing C, Nothwang HG, Vollmer M, Adolphs J, Hanusch H, Brandis M. A novel gene encoding an SH3 domain protein is mutated in nephronophthisis type 1. Nat Genet 1997;17:149-153.

3. Saunier S, Calado J, Heilig R, Silbermann F, Benessy F, Morin G, Konrad M, Broyer M, Gubler MC, Weissenbach J, Antignac C. A novel gene that encodes a protein with a putative src homology 3 domain is a candidate gene for familial juvenile nephronophthisis. Hum Mol Genet $1997 ; 6: 2317-2323$.

4. Donaldson JC, Dise RS, Ritchie MD, Hanks SK. Nephrocystinconserved domains involved in targeting to epithelial cellcell junctions, interaction with filamins, and establishing cell polarity. J Biol Chem 2002;277:29028-29035.

5. Otto EA, Schermer B, Obara T, O'Toole JF, Hiller KS, Mueller AM, Ruf RG, Hoefele J, Beekmann F, Landau D, Foreman JW, Goodship JA, Strachan T, Kispert A, Wolf MT, Gagnadoux MF, Nivet $\mathrm{H}$, Antignac C, Walz G, Drummond IA, Benzing T, Hildebrandt $\mathrm{F}$. Mutations in INVS encoding inversin cause nephronophthisis type 2, linking renal cystic disease to the function of primary cilia and left-right axis determination. Nat Genet 2003;34:413-420.

6. Mollet G, Salomon R, Gribouval O, Silbermann F, Bacq D, Landthaler G, Milford D, Nayir A, Rizzoni G, Antignac C, Saunier $S$. The gene mutated in juvenile nephronophthisis type 4 encodes a novel protein that interacts with nephrocystin. Nat Genet 2002;32:300-305.

7. Mollet G, Silbermann F, Delous F, Salomon R, Antignac C, Saunier $S$. Characterisation of the nephrocystin-4 complex and subcellular localization of nephrocystin-4 in primary cilia and in centrosomes. Submitted 2004.

8. Benzing T, Gerke P, Hopker K, Hildebrandt F, Kim E, Walz G. Nephrocystin interacts with Pyk2, p130(Cas), and tensin and 
triggers phosphorylation of Pyk2. Proc Natl Acad Sci U S A $2001 ; 98: 9784-9789$.

9. Saunier S, Calado J, Benessy F, Silbermann F, Heilig R, Weissenbach J, Antignac C. Characterization of the NPHP1 locus: mutational mechanism involved in deletions in familial juvenile nephronophthisis. Am J Hum Genet $2000 ; 66: 778-789$.

10. Wittekind M, Mueller L. HNCACB, a high sensitivity 3D NMR experiment to correlate amide proton and nitrogen resonances with the $\mathrm{a}$ and $\mathrm{b}$-carbon resonances in proteins. J Magn Reson $1993 ; 101$ (B) : 201-205.

11. Grzesiek S, Bax A. Correlating backbone amide and side chain resonances in larger proteins by multiple relayed triple resonance NMR. J Am Chem Soc 1992;114:6291-6293.

12. Vuister GW, Bax A. Quantitative J-correlation: a new approach for measuring homonuclear three-bond $\mathrm{J}(\mathrm{HN}-\mathrm{H} \alpha)$ couplings in ${ }^{15} \mathrm{~N}$-enriched proteins. J Am Chem SoC $1993 ; 115: 7772-7777$.

13. Archer SJ, Ikura M, Torchia DA, Bax A. An alternative 3D NMR technique for correlating backbone ${ }^{15} \mathrm{~N}$ with side chain $\mathrm{H}^{\beta}$ resonances in larger proteins. J Magn Reson 1991;95:636-641.

14. Güntert P, Braun W, Wüthrich K. Efficient computation of three-dimensional protein structures in solution from nuclear magnetic resonance data using the program DIANA and the upporting programs CALIBA, HABAS and GLOMSA. J Mol Biol $1991 ; 217: 517-530$.

15. Güntert $P$, Wüthrich $K$. Improved efficiency of protein structure calculations from NMR data using the program DIANA with redundant dihedral angle constraints. J Biomol NMR $1991 ; 1: 447-456$.

16. Brünger AT. X-PLOR Version 3.1. A system for X-Ray Crystallography and NMR. New Haven CT.: Yale University Press; 1992 .

17. Dardel F, Ragusa S, Lazennec C, Blanquet S, Meinnel T. Solution structure of nickel-peptide deformylase. J Mol Biol $1998 ; 280: 501-513$.

18. Bax A, Clore GM, Gronenborn AM. ${ }^{1} \mathrm{H}-{ }^{1} \mathrm{H}$ correlation via isotropic mixing of ${ }^{13} \mathrm{C}$ magnetization, a new three- 
dimensional approach for assigning ${ }^{1} \mathrm{H}$ and ${ }^{13} \mathrm{C}$ spectra of ${ }^{13} \mathrm{C}$ enriched proteins. J Magn Reson 1990;88:425-431.

19. Marion D, Driscoll PC, Kay LE, Wingfield PT, Bax A, Gronenborn AM, Clore GM. Overcoming the overlap problem in the assignment of ${ }^{1} \mathrm{H}$ NMR spectra of layer proteins HartmannHahn multiple quantum coherence and nuclear Overhauser multiple quantum coherence spectroscopy: Application to interleukin I $\beta$. Biochemistry 1989;28:6150-6156.

20. Zuiderweg ERP, Fesik SW. Heteronuclear three-dimensional NMR spectroscopy of the inflammatory protein C5a. Biochemistry 1989;28:2387-2391.

21. Wu X, Knudsen B, Feller SM, Zheng J, Sali A, Cowburn D, Hanafusa H, Kuriyan J. Structural basis for the specific interaction of lysine-containing proline-rich peptides with the N-terminal SH3 domain of C-Crk. Structure 1995;3:215226 .

22. Larson SM, Davidson AR. The identification of conserved interactions within the SH3 domain by alignment of sequences and structures. Protein Sci 2000;9:2170-2180.

23. Lim WA, Richards FM, FOX RO. Structural determinants of peptide-binding orientation and of sequence specificity in SH3 domains. Nature 1994;372:375-379.

24. Zamanian JL, Kelly RB. Intersectin $1 \mathrm{~L}$ guanine nucleotide exchange activity is regulated by adjacent src homology 3 domains that are also involved in endocytosis. Mol Biol Cell $2003 ; 14: 1624-1637$.

25. Bar-Sagi D, Rotin D, Batzer A, Mandiyan V, Schlessinger J. SH3 domains direct cellular localization of signaling molecules. Cell 1993;74:83-91.

26. Abram CL, Seals DF, Pass I, Salinsky D, Maurer L, Roth TM, Courtneidge SA. The adaptor protein fish associates with members of the ADAMs family and localizes to podosomes of Src-transformed cells. J Biol Chem 2003;278:16844-16851.

27. Nakamura H, Sudo T, Tsuiki H, Miyake H, Morisaki T, Sasaki J, Masuko N, Kochi M, Ushio Y, Saya H. Identification of a novel human homolog of the Drosophila dlg, P-dlg, specifically expressed in the gland tissues and interacting with p55. FEBS Lett 1998;433:63-67. 
28. Bauer F, Urdaci M, Aigle M, Crouzet M. Alteration of a yeast SH3 protein leads to conditional viability with defects in cytoskeletal and budding patterns. Mol Cell Biol $1993 ; 13: 5070-5084$.

29. Chen YJ, Lin SC, Tzeng SR, Patel HV, Lyu PC, Cheng JW. Stability and folding of the sH3 domain of Bruton's tyrosine kinase. Proteins 1996;26:465-471.

30. Grantcharova VP, Baker D. Folding dynamics of the src SH3 domain. Biochemistry 1997;36:15685-15692.

31. Plaxco KW, Guijarro JI, Morton CJ, Pitkeathly M, Campbell ID, Dobson CM. The folding kinetics and thermodynamics of the Fyn-SH3 domain. Biochemistry 1998;37:2529-2537.

32. Filimonov VV, Azuaga AI, Viguera AR, Serrano L, Mateo PL. A thermodynamic analysis of a family of small globular proteins: SH3 domains. Biophys Chem 1999;77:195-208.

33. Cobos ES, Filimonov VV, Vega MC, Mateo PL, Serrano L, Martinez JC. A thermodynamic and kinetic analysis of the folding pathway of an SH3 domain entropically stabilised by a redesigned hydrophobic core. J Mol Biol 2003;328:221-233.

34. Di Nardo AA, Larson SM, Davidson AR. The relationship between conservation, thermodynamic stability, and function in the SH3 domain hydrophobic core. J Mol Biol 2003;333:641655 .

35. Yi Q, Bystroff C, Rajagopal P, Klevit RE, Baker D. Prediction and structural characterization of an independently folding substructure in the src sH3 domain. J Mol Biol 1998;283:293-300.

36. Grantcharova VP, Riddle DS, Santiago JV, Baker D. Important role of hydrogen bonds in the structurally polarized transition state for folding of the src SH3 domain. Nat struct Biol 1998;5:714-720.

37. Pires JR, Hong X, Brockmann C, Volkmer-Engert R, Schneider-Mergener $J$, Oschkinat H, Erdmann R. The ScPex13p SH3 domain exposes two distinct binding sites for Pex5p and Pex14p. J Mol Biol 2003;326:1427-1435.

38. Koradi R, Billeter M, Wüthrich K. MOLMOL: A program for display and analysis of macromolecular structures. J Mol Graphics $1996 ; 14: 51-55$. 
Table I : Structural statistics

\begin{tabular}{lc}
\hline & Average \\
\hline Rms deviations from ideal geometry & $0.007 \AA$ \\
Bonds & $2.8 \mathrm{deg}$. \\
Angles & $0.3 \mathrm{deg}$. \\
Impropers & $-305 \mathrm{kcal} / \mathrm{mol}$ \\
$E_{\text {vaw }}$ & $94.4 \%$ \\
residues within allowed regions of & \\
the Ramachandran plot & \\
Rms deviations from experimental & \\
restraints & $0.04 \AA$ \\
NOE & $0.3 \AA$ \\
Largest violation & $1.3 \mathrm{deg}$. \\
Dihedrals & \\
Atomic rms differences : & \\
all structures vs mean structure & \\
backbone & $0.63 \AA$ \\
all heavy atoms & $1.09 \AA$ \\
backbone (core SH3) & $0.46 \AA$ \\
all heavy atoms (core SH3) & $0.88 \AA$ \\
\hline
\end{tabular}

The Van derWaals energies of the refined conformers were calculated with X-PLOR, using a cut-off of $7.5 \AA$. Average values of the energies and the r.m.s.d. were calculated over the set of 17 conformers (an overlay is shown in Figure 2). For structural comparisons, the core SH3 region, corresponded to residues 154-165, 169-194, 197-210. 
$150 \quad 160 \quad 170 \quad 180 \quad 190 \quad 200 \quad 210$ GSESHKWSTGEEYIAVGDFTAOQVGDLTFKKGEILLVIEKKPDGWWIAKDAKGNEGLVPRTYLEPYSE

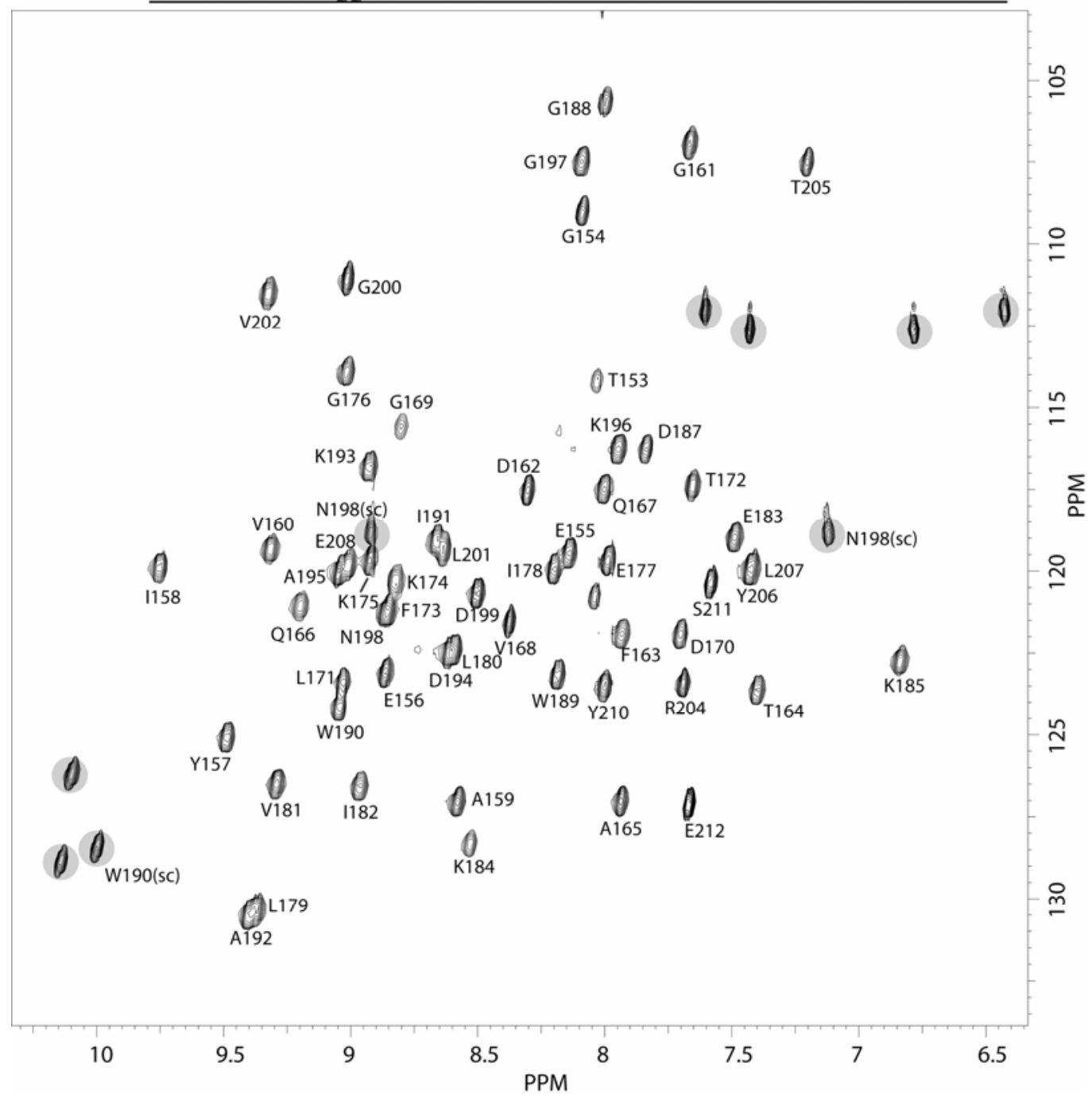

Figure 1 

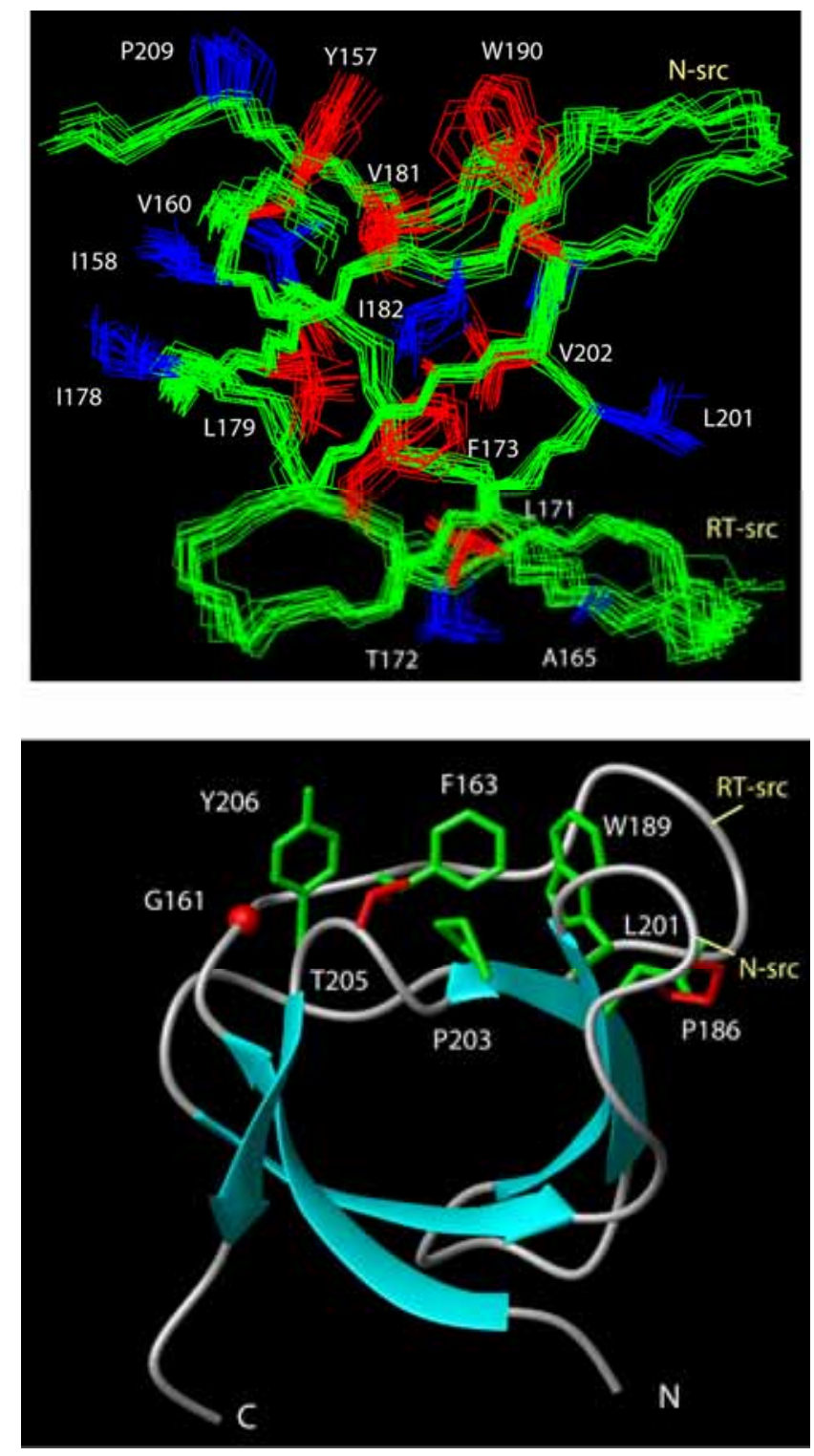

Figure 2 


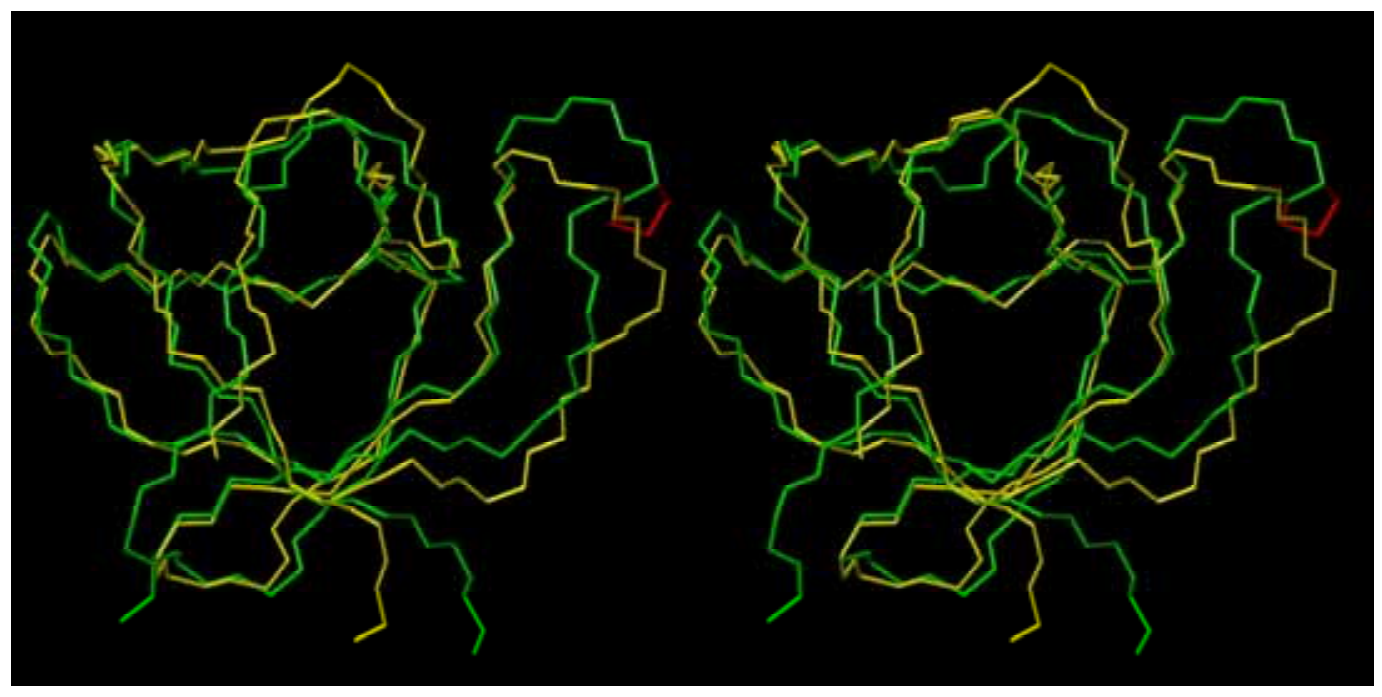

Figure 3 


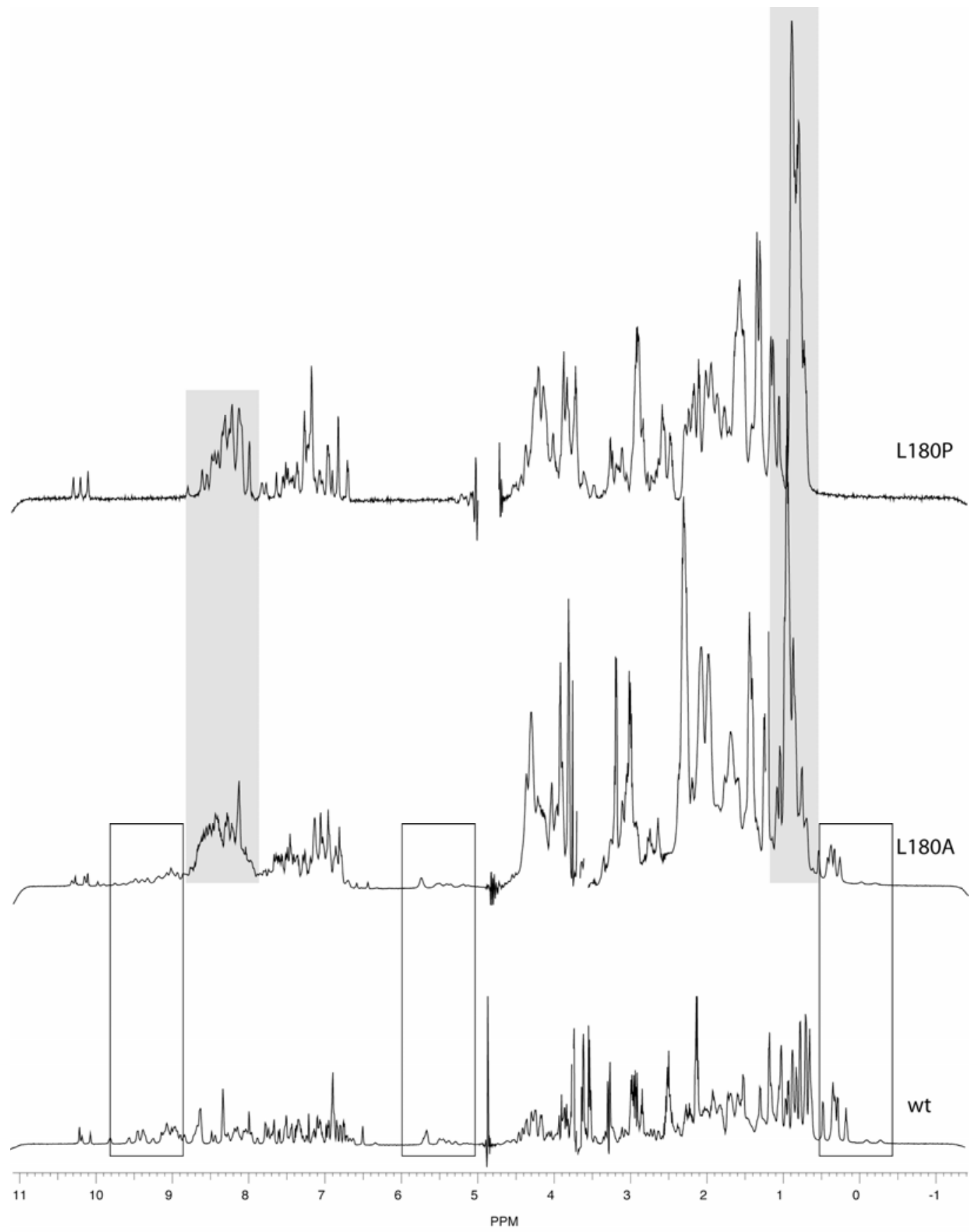

Figure 4 


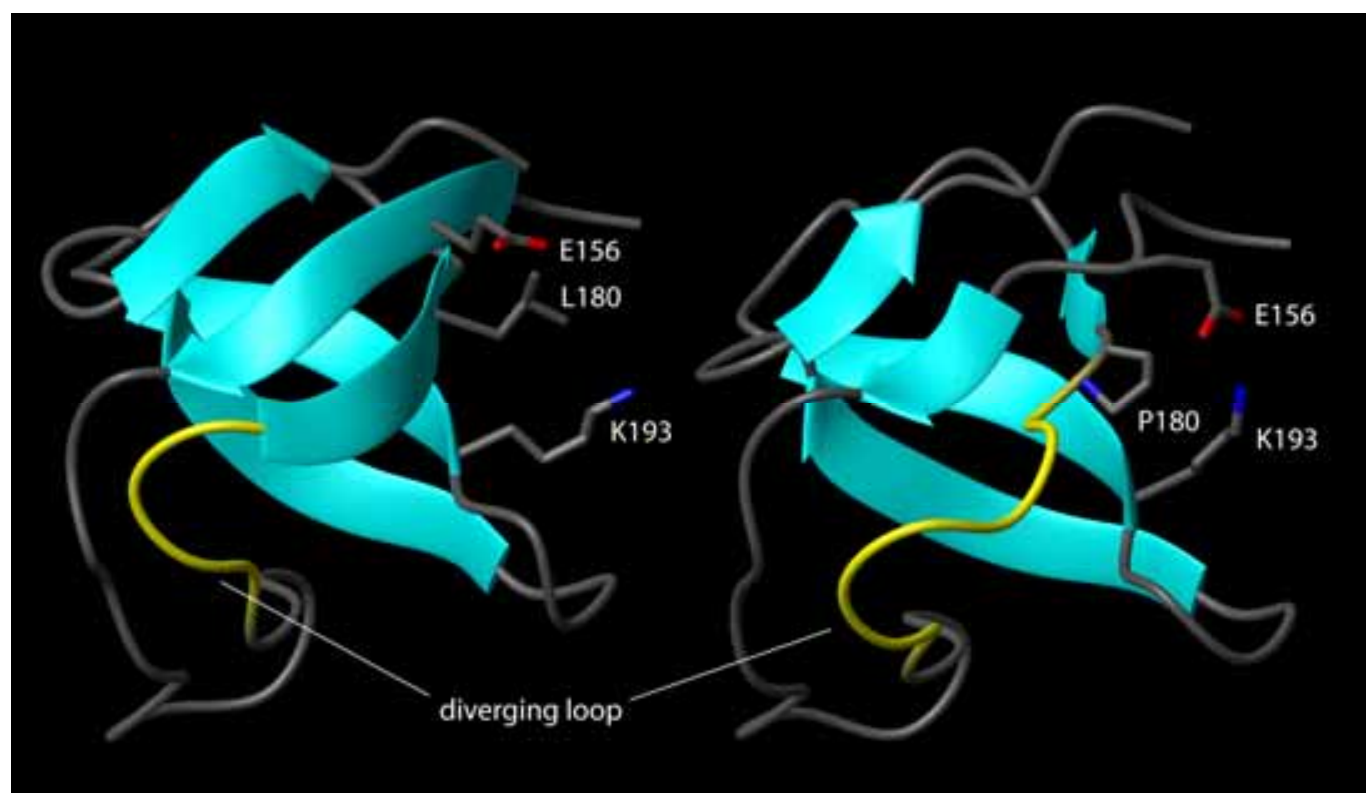

Figure 5 